\title{
INFLUENCE OF RIGHT VENTRICULAR FUNCTION ON THE DEVELOPMENT OF PRIMARY GRAFT DYSFUNCTION AFTER LUNG TRANSPLANTATION
}

Purificación Pérez-Terán $M D^{a, h}$, Oriol Roca $M D, P h D^{a, i}$, José Rodríguez-Palomares MD, $P h D^{b}$, Judit Sacanell $M D^{a}$, Sandra Leal $M D^{a}$, Joan Solé MD, PhD', María I. Rochera $M^{d}{ }^{d}$, Antonio Román $M D, P h^{e}$, Juan C. Ruiz-Rodríguez $M^{a}{ }^{a}$, Joaquim Gea $M D^{f, i j}, P h D$ Arturo Evangelista MD, $P h D^{b}$, Joan R. Masclans MD, PhD ${ }^{g, j}$.

${ }^{a}$ Critical Care, ${ }^{b}$ Cardiology, ${ }^{c}$ Thoracic Surgery, ${ }^{d}$ Anesthesiology and ${ }^{e}$ Respiratory Departments, Vall d'Hebron University Hospital, Institut de Recerca Vall d'Hebron (VHIR). ${ }^{f}$ Respiratory and ${ }^{9}$ Critical Care Department, Parc de Salut Mar de Barcelona, Institut Hospital del Mar d'Investigacions Mèdiques (IMIM). "Departament de Medicina. Universitat Autònoma Barcelona, Barcelona, Spain. 'Universitat Pompeu Fabra, Barcelona, Spain. ${ }^{\mathrm{j}}$ CibeRes (Ciber de Enfermedades Respiratorias), Instituto de Salud Carlos III, Madrid, Spain.

Corresponding author: Purificación Pérez-Terán, MD.

Critical Care Department, Vall d'Hebron University Hospital.

Pg. Vall d'Hebron 119-129, 08035, Barcelona, Spain.

E-mail: puperez@vhebron.net. 


\section{ABBREVIATIONS LIST}

BLS: Basal free wall longitudinal strain

ICU: Intensive Care Unit

iNO: Inhaled nitric oxide

LOS: Length of stay

LT: Lung transplant

LTr: Lung transplant recipients

PGD: Primary graft dysfunction

PGD3: Primary graft dysfunction grade 3

$\mathrm{PH}$ : Pulmonary hypertension

RV: Right ventricle

RVF: Right ventricular function

mPAP: Mean pulmonary arterial pressure

sPAP: Systolic pulmonary arterial pressure

TAPSE: Tricuspid annular plane systolic excursion

2D: Two-dimensional 


\section{ABSTRACT \\ Background}

Primary graft dysfunction (PGD) remains a significant cause of lung transplant (LT) postoperative morbidity and mortality. The underlying mechanisms of PGD development are not completely understood. The purpose of this study was to analyze the effect of right ventricular function (RVF) on PGD development.

\section{Methods}

A retrospective analysis of a prospectively assessed cohort was performed at a single institution between July 2010 and June 2013. The primary outcome was development of primary graft dysfunction grade 3 (PGD3). Conventional echocardiographic parameters and speckle-tracking echocardiography, performed during the pretransplant evaluation phase up to one year before surgery, were used to assess preoperative RVF.

\section{Results}

One hundred and twenty lung transplant recipients $(\mathrm{LTr})$ were included. Systolic pulmonary arterial pressure (sPAP) $(48 \pm 20$ vs. $41 \pm 18 \mathrm{mmHg} ; \mathrm{p}=0.048)$ and ischemia time $(349 \pm 73$ vs. $306 \pm 92 \mathrm{~min} ; \mathrm{p}<0.01)$ were higher in $\mathrm{LTr}$ who developed PGD3. Patients who developed PGD3 had better RVF estimated by basal free wall longitudinal strain (BLS) $(-24 \pm 9$ vs. $-20 \pm 6 \%$; $p=0.039)$, but they had longer Intensive Care Unit (ICU) length of stay (LOS) and mechanical ventilation, and higher 6-month mortality. BLS $\geq-21.5 \%$ was the cutoff that best identified patients developing PGD3 (AUROC $0.70[\mathrm{Cl} 95 \%$ 0.54-0.85], $\mathrm{p}=0.020$ ). In the multivariate analysis, a BLS $\geq$ $21.5 \%$ was an independent risk factor for PGD3 development (OR 4.56, [CI 95\% 1.2017.38], $p=0.026]$, even after adjusting for potential confounding.

\section{Conclusions}

A better RVF, as measured by BLS, is a risk factor for severe PGD. Careful preoperative RVF assessment using Speckle-tracking echocardiography may identify LTr with the highest risk of developing PGD. 
Key Words: Primary Graft Dysfunction, Lung Transplant, Right Ventricle, Speckletracking echocardiography. 


\section{INTRODUCTION}

Despite significant progress in recent decades, the morbidity associated with lung transplant $(L T)$ remains unacceptably high ${ }^{1,2,3}$. Primary graft dysfunction (PGD) is a form of acute respiratory distress syndrome that occurs in the first 72 hours after allograft reperfusion in lung transplant recipients $(\mathrm{LTr})^{4}$. It remains the most common early complication of LT and contributes significantly to patients' morbidity and mortality ${ }^{1,2,5,6,7}$. PGD is the end result of a series of hits occurring from the time of brain death to the time of lung reperfusion. Ischemia-reperfusion injury has been identified as its main cause ${ }^{8}$.

Preoperative pulmonary hypertension $(\mathrm{PH})$ is common among patients with respiratory diseases who are undergoing $\mathrm{LT}^{9}$ and is associated with worse outcomes ${ }^{10,11}$. One possible explanation for this link is $P G D^{12,13}$. In fact, a recent meta-analysis has identified primary pulmonary hypertension and mean pulmonary arterial pressure (mPAP) as significant risk factors for severe PGD development ${ }^{7}$. Several studies have analysed factors involved in the pathogenesis of PH and PGD in LTr, including a greater endothelial injury from hemodynamic forces, inherent abnormalities in coagulation or inflammation, platelet activation, and cell adhesion ${ }^{14,15}$. But its underlying mechanism is not completely understood. It has also been hypothesized that an exacerbation of ischemic-reperfusion lung injury, due to the hemodynamic forces caused by a "well-trained" hyperdynamic right ventricle (RV) contracting against a reduced pulmonary vascular resistance of the implanted lungs, may also play a role in PGD development ${ }^{16,17}$. To our knowledge, however, the role of RV in the development of PGD has not been clarified.

The assessment of right ventricular function (RVF) has become increasingly important in the management of patients with $\mathrm{PH}^{18,19}$. This may also be the case in $\mathrm{LTr}$. Quantification of RVF remains a challenge; because of its complex geometry, conventional two-dimensional (2D) echocardiography does not provide a 
comprehensive evaluation $^{20}$. Speckle-tracking echocardiography is a relatively new, non-invasive method to estimate RVF by means of the analysis of wall deformation. It has been widely applied to the left ventricle ${ }^{21,22}$. More recently, it has been shown to highlight changes in RVF, particularly in $\mathrm{PH}^{23,24}$. Furthermore, free wall 2D-Strain quantifies the longitudinal RVF, which is one of the main mechanisms of blood ejection from the $\mathrm{RV}^{25,26}$ and makes it possible to differentiate active from passive motion regardless of the Doppler angle $e^{26,27,28}$.

The main objective of this study is to analyse the possible role of RVF in severe PGD development. We hypothesized that pulmonary hyperflow generated by a "well-trained" $\mathrm{RV}$, facing reduced pulmonary vascular resistance after lung implantation, may generate a higher degree of pulmonary edema and hence may be a risk factor for PGD development. To assess this possibility we evaluated RVF using conventional echocardiography techniques and Speckle-tracking echocardiography. 


\section{METHODS}

\section{Study design}

A retrospective analysis of a prospectively assessed cohort was performed at a single institution (Vall d'Hebron University Hospital, Barcelona, Spain). All LTr admitted between July 2010 and June 2013 were included. Healthy subjects matched by age and sex were used as controls.

The study was approved by the Clinical Research Ethics Committee of Vall d'Hebron University Hospital (PR (AG) 144/2013). The need for informed consent was waived because of the non-interventional nature of the study.

\section{Study Population}

One hundred and eighty-two LTr were considered for inclusion. Sixty-two patients were excluded for lack of systolic pulmonary arterial pressure (SPAP) data (Figure 1). In addition to echocardiographic or cardiac catheterization SPAP data, patients' demographic data (gender, age and lung pathology) were recorded. Data regarding intraoperative multiple transfusion, use of inhaled nitric oxide (iNO), cardiopulmonary bypass and ischemic time were also collected.

As current guidelines stress the lack of normative data regarding $R V \operatorname{strain}^{20}$, twenty healthy volunteers recruited from the database of Vall d'Hebron Hospital Echocardiography Department and matched for age and sex were included as a control group.

\section{Definition of primary graft dysfunction}

The primary outcome was PGD grade 3 (PGD3) recorded during the first 72 hours post-surgery. In accordance with the report of the ISHLT Working Group on Primary Lung Graft Dysfunction PGD3 was defined as the presence of radiographic infiltrates consistent with pulmonary edema and a $\mathrm{PaO}_{2} / \mathrm{F}_{1} \mathrm{O}_{2}$ ratio less than $200 \mathrm{mmHg}^{4}$. The timepoints for the evaluation of PGD3 development were 6, 24, 48 and 72 hours after the start of graft perfusion ${ }^{4}$. The $\mathrm{PaO}_{2} / \mathrm{F}_{1} \mathrm{O}_{2}$ ratio was carefully measured in mechanically ventilated patients. Patients who were not intubated at the time of 
$\mathrm{PaO}_{2} / \mathrm{F}_{1} \mathrm{O}_{2}$ measurement were graded as no $\mathrm{PGD}^{4}$. In the single lung transplants, PGD3 development was determined by a $\mathrm{PaO}_{2} / \mathrm{F}_{1} \mathrm{O}_{2}$ ratio less than $200 \mathrm{mmHg}$ and the presence of radiographic infiltrates in the implanted lung ${ }^{4}$. Further, the researchers who graded PGD were blinded to the echocardiographic results and other clinical findings.

\section{Definition of pulmonary hypertension}

In accordance with European and American Guidelines, SPAP was measured by rightheart cardiac catheterization or alternatively by echocardiography ${ }^{29,30}$. In both cases, SPAP was obtained at the time of LT evaluation. SPAP echocardiography measures were attempted in all patients based on the modified Bernoulli equation: $s P A P=4 v^{2}+R i g h t$ atrial pressure, where $v$ is the peak velocity of the tricuspid regurgitation jet and the right atrial pressure was estimated by the collapsibility of the inferior vena cava on inspiration ${ }^{20}$.

\section{Echocardiography}

We collected data from echocardiograms performed by expert echocardiographers from the Cardiology Department during LT evaluation (acquired during the year before the $L T$ ). Routine digital gray scale 2D and tissue Doppler cine loops were obtained, in accordance with current guidelines ${ }^{31}$. RVF was evaluated from apical four-chamber images $^{20}$. After acquiring images, an expert echocardiographer performed the blinded offline study.

Conventional echocardiographic assessment of $\mathrm{RV}$ was performed using three different parameters. First, RV Fractional Area Change was obtained by tracing the RV endocardium in both systole and diastole, and was calculated as follows: RV Fractional Area Change $=(\mathrm{RV}$ end-diastolic area $-\mathrm{RV}$ end-systolic area $) / \mathrm{RV}$ end-diastolic area $\mathrm{x}$ $100 \%$. Secondly, the TAPSE was calculated as the total excursion of free wall tricuspid annulus from its highest position to the peak descent during ventricular systole by 
means of the M-mode. Finally, the Doppler-derived tricuspid lateral annular systolic velocity was measured by the peak systolic velocity of the lateral corner of the tricuspid annulus ${ }^{20}$.

Speckle-tracking echocardiography of RV free wall was performed using a routine gray scale modified apical four-chamber view ${ }^{20}$. The measurements were performed offline with dedicated software (EchoPAC version BTO8; GE Vingmed Ultrasound). The RV free wall was divided into three standard segments (basal, middle, and apical), and then time-strain curves were generated. We evaluated only the RV free wall because previous studies have demonstrated a good correlation between free wall RV 2DStrain, RV ejection and RV end-systolic volume measured using cardiac magnetic resonance ${ }^{20,23,26,27}$. Longitudinal Strain and Strain Rate data were collected. Longitudinal Strain measures the percentage of myocardial deformation. As the RV contracts, the muscle shortens in the longitudinal and circumferential dimensions (negative strain). Strain rate measures the time course of deformation.

\section{Outcome measures}

The primary outcome was to study the relationship between PGD3 development and preoperative RVF.

Secondary outcomes analysed were other possible risk factors for PGD3 development, morbidity and mortality, as well as the characterization of LTr RVF changes by conventional ultrasound methods and 2D-Strain.

\section{Statistical analysis}

The statistical analysis was performed with SPSS (release 18.0, SPSS Inc., Chicago, Illinois, USA). Baseline characteristics were expressed as mean (standard deviation) for continuous variables and frequencies (percentage) for categorical variables. Comparisons between groups were made with the Chi-square or Fisher's test for 
categorical variables, and analysis of the variance (ANOVA) or student " $t$ " test for continuous variables. Differences in these variables were assessed according to PGD3 development. Comparisons were considered significant in the presence of a $P$ value $<0.05$. Discrimination of significant variables was tested by calculating the area of receiver operating characteristic curve (ROC). Multivariate logistic regression was used to identify which variables were independently associated with PGD3 development. Variables with $p<0.1$ in the univariate analysis were introduced into the multivariate model. Finally, we assessed the effect of all potential confounding variables on the variables that were independently associated with the development of PGD3. Confounding was defined as a change in the OR of $15 \%$ or greater on adjustment. In order to prevent model overfitting, we introduced all potential confounding variables one at time. 


\section{RESULTS}

One hundred and twenty LTr were included, of whom 46\% developed PGD3 in the first 72 hours of the postoperative period (Figure 1). Table 1 shows the preoperative, intraoperative and postoperative characteristics as well as outcomes of LTr included in our study. Patients with PGD3 presented higher preoperative sPAP $(48 \pm 20$ vs. $41 \pm 18$ $\mathrm{mmHg} ; \mathrm{p}=0.048)$, a longer ischemic time $(349 \pm 73$ vs. $306 \pm 92 \mathrm{~min} ; \mathrm{p}=0.005)$ and a higher use of intraoperative iNO (63 vs. 19\%; $p<0.001$ ). Patients who developed PGD3 in the early postoperative period had a higher Intensive Care Unit (ICU) length of stay (LOS) $(40 \pm 35$ vs. $25 \pm 35$ days; $p=0.032)$, mechanical ventilation length $(34 \pm 35$ vs. $20 \pm 34$ days; $p=0.037)$ and also higher ICU (16 vs. $3 \% ; p=0.023)$, 90 -day (14 vs. $3 \%$; $p=0.044)$ and 6-month mortality ( 25 vs. $8 \% ; p=0.027)$.

Compared with healthy volunteers, LTr presented a worse RVF (Table 2). However, basal longitudinal strain (BLS) was higher (better RVF) in LTr who developed PGD3 ($24 \pm 9$ vs. $-20 \pm 6 \% ; p=0.039$ ) (Table 3 ). $B L S \geq-21.5 \%$ was the cutoff that best identified patients developing PGD3 (AUROC 0.70 [Cl 95\% 0.54-0.85], sensitivity 68\%, specificity 64\%, PPV $62 \%$ and NPV $70 \% ; p=0.020$ ). However, the positive likehood ratio was 2.45 . This means that, for a pre-test PGD3 incidence of $46 \%$, the incidence would be $31 \%$ if BLS is $<-21.5 \%$ and may increase until $61 \%$ if BLS is $\geq-21.5 \%$. Therefore, a BLS $\geq-21.5 \%$ increases the probability of PGD3 by $30 \%$. All variables with $p<0.1$ in the univariate analysis were included in a multivariate model to identify risk factors independently associated with PGD3 development. iNO was not included because in all probability it was used mainly to treat intraoperative hypoxemia secondary to appearance of PGD, and might therefore be considered as a consequence rather than a risk factor. $\mathrm{A} B L S \geq-21.5 \%$ was the only variable independently associated with PGD3 development (OR 4.56, [Cl 95\% 1.20-17.38], $\mathrm{p}=0.026$ ) (Table 4). Finally, in order to assess the effect of potential confounding, several models were also constructed including BLS (as the independent risk factor of PGD3) and other potential confounding variables (Table 5). No effect of confounding 
variables on BLS was observed and the effect of BLS on PGD3 development was significant at all times. 


\section{DISCUSSION}

This is the first study to evaluate the role of RVF in the pathogenesis of PGD. Two important results should be highlighted. First, PGD had an important effect on both short and long-term morbidity and mortality. Second, we demonstrate that a better RVF, measured by BLS, is an independent risk factor for PGD3 development even after adjusting for potential confounding. This result has not been published previously and provides new evidence to further our understanding of PGD pathophysiology.

The incidence of PGD3 varies between $12 \%$ and $32 \%$ depending on the time point chosen for grading ${ }^{1,2,7,13,32}$. A PGD3 incidence of $46 \%$ results from selecting the most severe grade recorded during the 72-hour period. However, 72-hour PGD3 incidence is around $17 \%$. But the point is that PGD remains a significant cause of posttransplantation morbidity and mortality. Reported 30-day mortality rates of patients who develop PGD are nearly eight times higher; PGD also leads to increased length of mechanical ventilation and ICU stay, increased perioperative complications and poor functional outcomes ${ }^{1,7}$.

Despite the significant morbidity and mortality in patients who develop PGD, the associated risk factors remain controversial. A recent meta-analysis showed that female gender, preoperative diagnosis of pulmonary fibrosis or primary $\mathrm{PH}$, use of cardiopulmonary bypass, multiple transfusions and mPAP were significantly and consistently associated with development of $\mathrm{PGD}^{7}$. Our study observed no differences in gender, preoperative diagnosis, use of CPB or multiple transfusions; however, we found that a higher ischemic time is related to the development of $\mathrm{PGD}^{8,13}$. Intraoperative use of iNO was more frequent in patients with PGD: this should not be considered a risk factor for PGD but a consequence of the appearance of intraoperative hypoxemia ${ }^{7,8}$. However, we cannot rule out the possible role of iNO in decreasing RV afterload and enhancing RVF.

Primary $\mathrm{PH}$ is the most significant risk factor for $P G D^{11,14,34}$. As we pointed out above, the results of a recent meta-analysis showed strong associations between primary $\mathrm{PH}$ 
and mPAP with PGD ${ }^{8}$. We also observed an association between SPAP and PGD3. However, the reason for it is not fully understood. Christie et $\mathrm{al}^{12}$ showed that the diagnosis of primary $\mathrm{PH}$ was even more strongly associated with an increased of risk of PGD after adjustment for recipient PAP. This suggests that factors other than the absolute value of PAP may be important in the pathogenesis of PGD. It should be noted that immediately after lung implantation RV afterload is acutely reduced, leading to an increase in pulmonary blood flow and therefore a greater shear stress, which causes capillary leak and impairs graft function $8,14,15$. Thus, PAP, or perhaps right cardiac output, during the initial 10 minutes after lung reperfusion is of prime importance because a rapid perfusion after a period of ischemia may exacerbate the existing ischemia-reperfusion injury and increase pulmonary edema generation ${ }^{33,34}$. It is also well known that patients with pulmonary diseases and $\mathrm{PH}$ can also present a secondary RV dysfunction. This myocardial involvement may be variable and some patients may present a hyperdynamic or compensatory response preceding the development of RV dysfunction ${ }^{24}$. Thus, LTr with a normal or hyperdynamic RV will have a higher pulmonary blood flow after pulmonary implantation. For this reason, RVF can be also considered as a possible risk factor of PGD. We hypothesized that, regardless of PAP, patients with better RVF may present a greater pulmonary flow after lung implantation and consequently a higher risk of PGD.

However, quantification of RVF remains a challenge because of its complex geometry ${ }^{20}$. Conventional 2D-echocardiography does not allow a comprehensive evaluation because of the right ventricle's complex crescent-shaped structure, wrapped around the left ventricle ${ }^{31}$. Recently, Speckle-tracking echocardiography has been introduced for the evaluation of global and regional RVF. Speckle-tracking assesses myocardial deformation without being limited by the Doppler beam angle ${ }^{27}$, allows us to differentiate active from passive motion ${ }^{28}$ and is more effective for evaluating subtle changes than other conventional echocardiographic techniques. Further, Speckletracking has a good intra and interobserver agreement, with low bias ${ }^{23,24}$. In this regard, 
$\mathrm{RV}$ free wall longitudinal Strain quantifies the longitudinal RV systolic function, which is one of the main mechanisms of blood ejection from $\mathrm{RV}^{25}$ and correlates significantly with the RV ejection fraction measured using cardiac magnetic resonance re,23,26. $^{20}$. However, in patients with $\mathrm{PH}$, previous studies have demonstrated that RV pressure overload reduces global and regional $\mathrm{RV}$ strain and increases regional heterogeneity ${ }^{18,26,27,35}$. Due to the progressive nature of the disease, the apical segments are the most affected; they are then compensated for by the mid and basal $\mathrm{RV}$ region, which becomes the main determinant of the $\mathrm{RV}$ cardiac output ${ }^{36}$. Therefore, in these patients the basal level segment is an accurate and reproducible estimator of $\mathrm{RVF}^{37}$.

In our study, comparing the RVF of the control group and LTr we observed decreases in conventional echocardiography parameters (except Doppler-derived tricuspid lateral annular systolic velocity) and longitudinal Strain, but not in Strain rate. However, it is important to consider that (with the exception of apical longitudinal Strain) all echocardiography parameters are within normal range ${ }^{20}$. However, analysing the RVF of LTr who developed PGD3, we observed that they have a higher BLS, meaning that LTr with better RVF have a higher prevalence of PGD3. Furthermore, patients with a BLS $\geq-21.5 \%$ have an increased risk of developing severe PGD. These results argue in favor of performing a Speckle-tracking echocardiography study in $\mathrm{LTr}$ echocardiography evaluation to identify patients with higher risk of developing PGD3. These findings are especially important for two reasons. First, they provide further evidence of the pathophysiology of PGD. Second, they might allow an assessment of the effectiveness of new potential treatment strategies, such as the progressive intraoperative reintroduction of pulmonary flow, in patients with a high risk of PGD development in whom these strategies could provide more benefits in terms of pulmonary edema reduction ${ }^{16,33,34}$.

This study has some limitations. The first is that, due to its retrospective nature, donor characteristics were not available and the time between echocardiographic evaluation 
and LT was variable. Moreover, even though more than 100 patients were included, the possibility of a beta-type error cannot be entirely ruled out. Therefore the results obtained need to be confirmed in a prospective study in which echocardiography is evaluated immediately before transplant. Second, the speckle-tracking technique involves manual tracing of the endocardial border, which requires special care and may be particularly difficult in the apical regions of the free wall. Finally, not all sPAP data were recorded from RHC; some of them were obtained by means of echocardiography. However, in the 32 patients who had catheterization and echocardiography sPAP data, mean catheterization SPAP was $46 \mathrm{mmHg}(+-25)$ and mean echocardiography sPAP $50 \mathrm{mmHg}(+-26)(p=0.148)$, with a correlation coefficient of $0.77(p<0.01)$. Therefore, the two methods produced broadly similar clinical results.

Nevertheless, despite these potential limitations, this is the first study to demonstrate the importance of RVF in the appearance of PGD. We believe that the results provide new evidence regarding the pathophysiology of PGD, which is the most common early complication of LT and contributes significantly to patient morbidity and mortality. Understanding the mechanisms that lead to PGD is vital for designing therapeutic strategies focused on PGD prevention that can improve outcomes in LTr patients.

In summary, the present study is the first to demonstrate that RVF, measured by BLS, is an independent risk factor for severe PGD development. Therefore, accurate preoperative RVF assessment by means of $2 D$-Strain is essential to identify $L T r$ at a higher risk of PGD development who would benefit the most from an extremely careful perioperative management strategy able to limit pulmonary overflow. 


\section{GRANT ACKNOWLEDGEMENTS}

Dr. Pérez-Terán is granted by a "Rio Hortega" grant (Ref. CM12/00216) from the Instituto de Salud Carlos III, Ministerio de Ciencia e Innovación. 


\section{REFERENCES}

1. Christie JD, Sager JS, Kimmel SE, et al. Impact of primary graft failure on outcomes following lung transplantation. Chest. 2005;127(1):161-5.

2. Christie JD, Kotloff RM, Ahya VN, et al. The effect of primary graft dysfunction on survival after lung transplantation. Am $J$ Respir Crit Care Med. 2005;171(11):1312-6.

3. Leal S, Sacanell J, Riera J, et al. Early postoperative management of lung transplantation. Minerva Anestesiol. 2014. In press.

4. Christie JD, Carby M, Bag R, et al. Report of the ISHLT Working Group on Primary Lung Graft Dysfunction part II: definition. A consensus statement of the International Society for Heart and Lung Transplantation. J Heart Lung Transplant. 2005;24(10):1454-9.

5. Prekker ME, Nath DS, Walker AR, et al. Validation of the Proposed International Society for Heart and Lung Transplantation Grading System for Primary Graft Dysfunction After Lung Transplantation. J Heart Lung Transplant. 2006;25(4):371-8.

6. Arcasoy SM, Fisher A, Hachem RR, et al. Report of the ISHLT Working Group on Primary Lung Graft Dysfunction part V: predictors and outcomes. J Heart Lung Transplant. 2005;24(10):1483-8.

7. Liu Y, Liu Y, Su L, et al. Recipient-related clinical risk factors for primary graft dysfunction after lung transplantation: a systematic review and meta-analysis. PLoS One. 2014;9(3):e92773.

8. De Perrot M, Liu M, Waddell TK, et al. Ischemia-reperfusion-induced lung injury. Am J Respir Crit Care Med. 2003;167(4):490-511. 
9. Shorr a F, Wainright JL, Cors CS, et al. Pulmonary hypertension in patients with pulmonary fibrosis awaiting lung transplant. Eur Respir J. 2007;30(4):715-21.

10. Barr ML, Kawut SM, Whelan TP, et al. Report of the ISHLT Working Group on Primary Lung Graft Dysfunction part IV: recipient-related risk factors and markers. J Heart Lung Transplant. 2005;24(10):1468-82.

11. Whelan TPM, Dunitz JM, Kelly RF, et al. Effect of preoperative pulmonary artery pressure on early survival after lung transplantation for idiopathic pulmonary fibrosis. J Heart Lung Transplant. 2005;24(9):1269-74.

12. Christie JD, Kotloff RM, Pochettino A, et al. Clinical risk factors for primary graft failure following lung transplantation. Chest. 2003;124(4):1232-41.

13. Whitson BA, Nath DS, Johnson AC, et al. Risk factors for primary graft dysfunction after lung transplantation. J Thorac Cardiovasc Surg. 2006;131(1):73-80.

14. Christie JD, Robinson N, Ware LB, et al. Association of protein C and type 1 plasminogen activator inhibitor with primary graft dysfunction. Am J Respir Crit Care Med. 2007;175(1):69-74.

15. Covarrubias M, Ware LB, Kawut SM, et al. Plasma intercellular adhesion molecule-1 and von Willebrand factor in primary graft dysfunction after lung transplantation. Am J Transplant. 2007;7(11):2573-8.

16. De Perrot M, Liu M, Waddell TK, Keshavjee S. Ischemia-reperfusion-induced lung injury. Am J Respir Crit Care Med. 2003;167(4):490-511.

17. Wrobel JP, Thompson BR, Snell GI, et al. Preoperative echocardiographicdefined moderate-severe pulmonary hypertension predicts prolonged duration of 
mechanical ventilation following lung transplantation for patients with COPD. Lung. 2012;190(6):635-43.

18. Pirat B, McCulloch ML, Zoghbi W. Evaluation of global and regional right ventricular systolic function in patients with pulmonary hypertension using a novel speckle tracking method. Am J Cardiol. 2006;98(5):699-704.

19. Grapsa J, Dawson D, Nihoyannopoulos P. Assessment of right ventricular structure and function in pulmonary hypertension. J Cardiovasc Ultrasound. 2011;19(3):115-25.

20. Rudski LG, Lai WW, Afilalo J, et al. Guidelines for the echocardiographic assessment of the right heart in adults: a report from the American Society of Echocardiography endorsed by the European Association of Echocardiography, a registered branch of the European Society of Cardiology, and t. J Am Soc Echocardiogr. 2010;23(7):685-713.

21. Marwick TH. Measurement of strain and strain rate by echocardiography: ready for prime time? J Am Coll Cardiol. 2006;47(7):1313-27.

22. Bussadori $\mathrm{C}$, Moreo a, Di Donato $\mathrm{M}$, et al. A new 2D-based method for myocardial velocity strain and strain rate quantification in a normal adult and paediatric population: assessment of reference values. Cardiovasc Ultrasound. $2009 ; 7: 8$

23. Motoji $\mathrm{Y}$, Tanaka H, Fukuda $\mathrm{Y}$, et al. Efficacy of Right Ventricular Free-Wall Longitudinal Speckle-Tracking Strain for Predicting Long-Term Outcome in Patients With Pulmonary Hypertension. Circ J. 2013;77(3):756-763.

24. Matias C, Isla LP De, Vasconcelos M, et al. Speckle-tracking-derived strain and strain-rate analysis: a technique for the evaluation of early alterations in right 
ventricle systolic function in patients with systemic sclerosis and normal pulmonary artery pressure. J Cardiovasc Med (Hagerstown). 2009;10(2):129-34.

25. Rushmer RF, Crystal DK. Changes in Configuration of the Ventricular Chambers during the Cardiac Cycle. Circulation. 1951;4(2):211-218.

26. Fukuda $\mathrm{Y}$, Tanaka $\mathrm{H}$, Sugiyama $\mathrm{D}$, et al. Utility of right ventricular free wall speckle-tracking strain for evaluation of right ventricular performance in patients with pulmonary hypertension. J Am Soc Echocardiogr. 2011;24(10):1101-8.

27. Meris A, Faletra $F$, Conca $C$, et al. Timing and magnitude of regional right ventricular function: a speckle tracking-derived strain study of normal subjects and patients with right ventricular dysfunction. J Am Soc Echocardiogr. 2010;23(8):823-31.

28. Tanaka H, Nesser H-J, Buck $\mathrm{T}$, et al. Dyssynchrony by speckle-tracking echocardiography and response to cardiac resynchronization therapy: results of the Speckle Tracking and Resynchronization (STAR) study. Eur Heart J. 2010;31(14):1690-700.

29. Galiè N, Hoeper MM, Humbert $M$, et al. Guidelines for the diagnosis and treatment of pulmonary hypertension: the Task Force for the Diagnosis and Treatment of Pulmonary Hypertension of the European Society of Cardiology (ESC) and the European Respiratory Society (ERS), endorsed by the Internat. Eur Heart J. 2009;30(20):2493-537.

30. McLaughlin V V, Archer SL, Badesch DB, et al. ACCF/AHA 2009 expert consensus document on pulmonary hypertension: a report of the American College of Cardiology Foundation Task Force on Expert Consensus Documents and the American Heart Association: developed in collaboration with the American College. Circulation. 2009;119(16):2250-94. 
31. Lang RM, Bierig $M$, Devereux RB, et al. Recommendations for chamber quantification: a report from the American Society of Echocardiography's Guidelines and Standards Committee and the Chamber Quantification Writing Group, developed in conjunction with the European Association of Echocardiograph. J Am Soc Echocardiogr. 2005;18(12):1440-63.

32. Diamond JM, Lee JC, Kawut SM, et al. Clinical Risk Factors for Primary Graft Dysfunction after Lung Transplantation. Am $J$ Respir Crit Care Med. 2013;187(5):527-34.

33. Pierre AF, DeCampos KN, Liu M, et al. Rapid reperfusion causes stress failure in ischemic rat lungs. J Thorac Cardiovasc Surg. 1998;116(6):932-42.

34. Bhabra MS, Hopkinson DN, Shaw TE, et al. Controlled reperfusion protects lung grafts during a transient early increase in permeability. Ann Thorac Surg. 1998;65(1):187-92.

35. Leung DY, $\mathrm{Ng} \mathrm{ACT}$. Emerging clinical role of strain imaging in echocardiography. Heart Lung Circ. 2010;19(3):161-74. 6.

36. Calcutteea $A$, Lindqvist $P$, Soderberg $S$, et al. Global and regional right ventricular dysfunction in pulmonary hypertension. Echocardiography. 2014;31(2):164-71.

37. Vitarelli A, Conde $\mathrm{Y}$, Cimino $\mathrm{E}$, et al. Assessment of right ventricular function by strain rate imaging in chronic obstructive pulmonary disease. Eur Respir J. 2006;27(2):268-75. 
Figure 1. Recruitment flowchart.

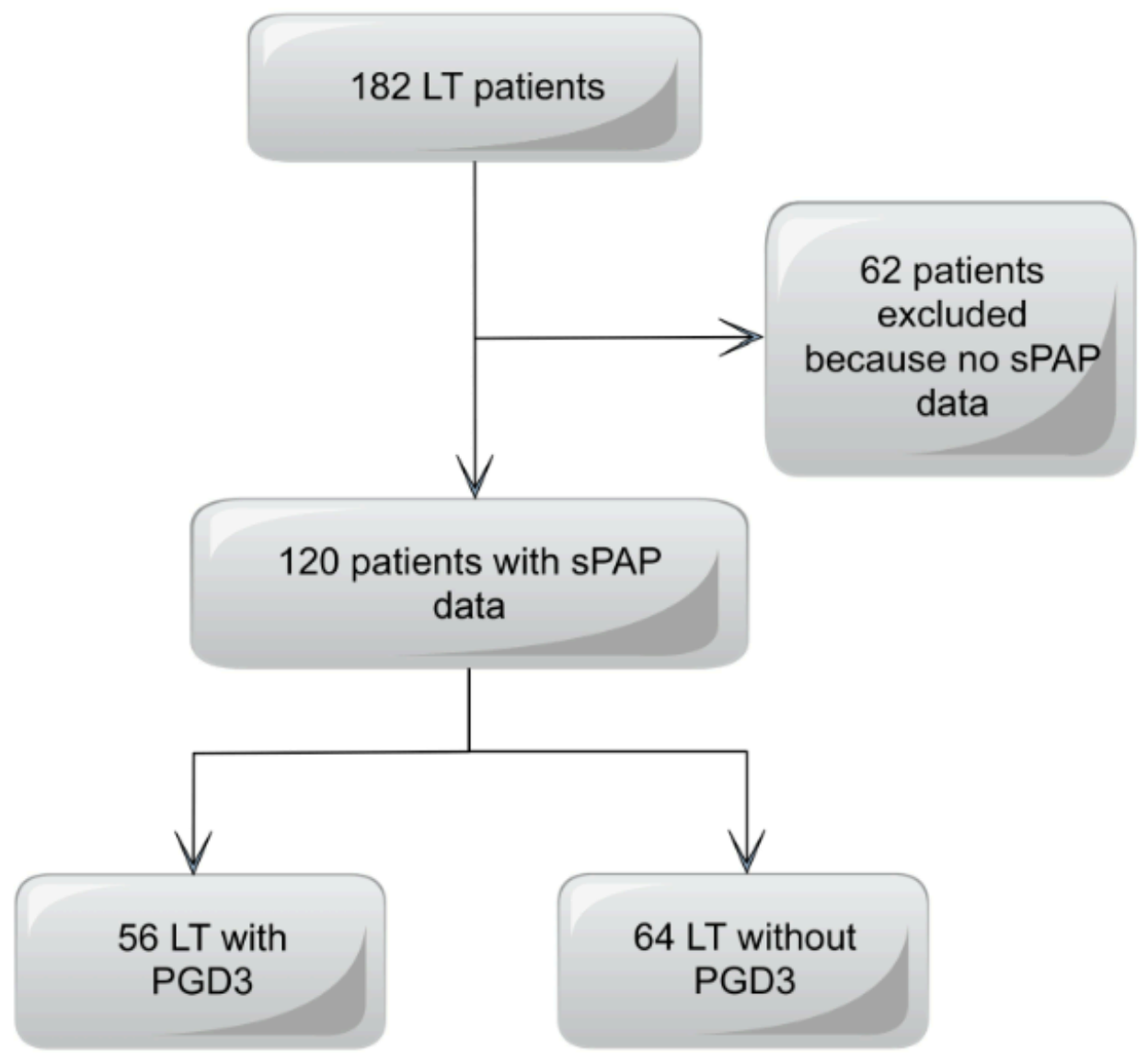

LT: Lung Transplant, sPAP: Systolic Pulmonary Arterial Pressure, PGD3: Pulmonary Graft Dysfunction Grade 3. 
Table 1. Lung Transplant Recipient Characteristics vs. PGD3

\begin{tabular}{|c|c|c|c|c|c|}
\hline & $\begin{array}{c}\text { No PGD3 } \\
(n=64)\end{array}$ & $\begin{array}{l}\text { PGD3 } \\
(n=56)\end{array}$ & OR & $95 \% \mathrm{Cl}$ & p-value \\
\hline \multicolumn{6}{|c|}{ PREOPERATIVE CHARACTERISTICS } \\
\hline Age (years) & $55(11)$ & $51(11)$ & 0.97 & $(0.94-1.00)$ & 0.065 \\
\hline Gender: Male & $36(56 \%)$ & $36(64 \%)$ & 0.71 & $(0.34-1.49)$ & 0.456 \\
\hline BMI $\quad\left(\mathrm{kg} / \mathrm{m}^{2}\right)$ & $26(8)$ & $27(5)$ & 0.27 & $(-4.17-2.65)$ & 0.658 \\
\hline Etiology & & & & & 0.697 \\
\hline - $\quad$ Pulmonary fibrosis & $18(28 \%)$ & $10(36 \%)$ & 1.42 & $(0.66-3.07)$ & \\
\hline - COPD & $34(53 \%)$ & $23(41 \%)$ & 0.58 & $(0.28-1.19)$ & \\
\hline - Pulmonary hypertension & $5(8 \%)$ & $10(9 \%)$ & 1.16 & $(0.32-4.22)$ & \\
\hline - Cystic fibrosis & $3(5 \%)$ & $2(4 \%)$ & 0.56 & $(0.50-6.39)$ & \\
\hline - Others & $4(6 \%)$ & $6(11 \%)$ & 1.76 & $(0.28-10.90)$ & \\
\hline sPAP $\quad(\mathrm{mmHg})$ & $41(18)$ & $48(20)$ & 1.06 & $(1.01-1.13)$ & 0.048 \\
\hline
\end{tabular}

INTRAOPERATIVE CHARACTERISTICS

Procedure type:

0.098

-Single lung transplant

$33(52 \%)$

$20(36 \%)$

$0.52 \quad(0.25-1.09)$

-Bilateral lung transplant

$41(48 \%)$

$26(64 \%)$

1.91

(0.02-3.99)

CPB use

$14(22 \%)$

$20(30 \%)$

0.96

$(0.37-2.52)$

0.305

iNO use

$12(19 \%)$

$35(63 \%)$

7.22

$(3.15-16.54)$

$<0.001$

Multiple transfusions

$19(30 \%)$

$17(40 \%)$

1.54

(0.72-3.31)

0.333

Ischemic time (min)

306 (92)

349 (73)

1.01

$(1.00-1.01)$

0.005

\section{POSTOPERATIVE CHARACTERISTICS}

$\begin{array}{lccccc}\text { ICU mortality } & 2(3 \%) & 9(16 \%) & 5.95 & (1.23-28.77) & 0.023 \\ \mathbf{2 8} \text { days mortality } & 1(2 \%) & 5(9 \%) & 6.18 & (0.67-54.56) & 0.096 \\ \mathbf{9 0} \text { days mortality } & 2(3 \%) & 8(14 \%) & 5.17 & (1.05-25.45) & 0.044 \\ \mathbf{6} \text { months mortality } & 4(8 \%) & 12(25 \%) & 4.08 & (1.22-13.70) & 0.027 \\ \text { ICU LOS (days) } & 25(35) & 40(35) & 1.01 & (1.00-1.02) & 0.032 \\ \text { MV length (days) } & 20(34) & 34(35) & 1.01 & (1.00-1.02) & 0.037\end{array}$

OR: Odds Ratio; Cl: Confidence Interval; sPAP: Systolic Pulmonary Arterial Pressure; COPD: Chronic Obstructive Pulmonary Disease; CPB: Cardiopulmonary Bypass, iNO: inhaled nitric oxide; PGD: Primary Graft Dysfunction; ICU: Intensive Care Unit; LOS: length of stay; MV: Mechanical Ventilation. 
Table 2. RV function: Controls vs. Lung transplant recipients.

\begin{tabular}{|c|c|c|c|}
\hline & $\begin{array}{l}\text { Control Group } \\
\qquad(n=20)\end{array}$ & $\begin{array}{c}\mathrm{LTr} \\
(n=120)\end{array}$ & p-value \\
\hline FAC (\%) & $50(9)$ & $38(10)$ & $<0.001$ \\
\hline TAPSE (mm) & $22(3)$ & $20(4)$ & 0.032 \\
\hline $\mathbf{S}^{\prime}(\mathrm{cm} / \mathrm{s})$ & $13.0(1.1)$ & $12.0(3.0)$ & 0.189 \\
\hline \multicolumn{4}{|l|}{ LS (\%) } \\
\hline Free Wall & $-25(5)$ & $-18(6)$ & $<0.001$ \\
\hline Basal & $-31(9)$ & $-22(8)$ & $<0.001$ \\
\hline Medium & $-27(6)$ & $-19(6)$ & $<0.001$ \\
\hline Apical & $-18(6)$ & $-13(6)$ & 0.002 \\
\hline \multicolumn{4}{|l|}{$\operatorname{Sr}\left(s^{-1}\right)$} \\
\hline Basal & $2.53(0.82)$ & $2.15(0.94)$ & 0.120 \\
\hline Medium & $1.66(0.41)$ & $1.54(0.72)$ & 0.497 \\
\hline Apical & $1.22(0.35)$ & $1.29(0.52)$ & 0.590 \\
\hline
\end{tabular}

FAC: Fractional Area Change, TAPSE: Tricuspid Annular Plane Systolic Excursion, S': Doppler-derived tricuspid lateral annular systolic velocity, LS: Longitudinal Strain, Sr: Strain Rate. 
Table 3. RV function: Lung transplant recipients vs. PGD3.

\begin{tabular}{|c|c|c|c|c|c|c|}
\hline & LRV & $\begin{array}{l}\text { No PGD3 } \\
(n=64)\end{array}$ & $\begin{array}{l}\text { PGD3 } \\
(n=56)\end{array}$ & OR & CI $95 \%$ & $p$-value \\
\hline FAC $(\%)$ & 35 & $37(9)$ & $40(11)$ & 1.03 & $(0.97-1.09)$ & 0.353 \\
\hline TAPSE (mm) & 16 & $21(4)$ & $20(4)$ & 0.95 & $(0.87-1.05)$ & 0.316 \\
\hline $\mathbf{S}^{\prime}(\mathrm{cm} / \mathrm{s})$ & 10 & $12.0(3.0)$ & $12.0(3.5)$ & 1.01 & $(0.84-1.22)$ & 0.913 \\
\hline \multicolumn{7}{|l|}{ LS (\%) } \\
\hline Free Wall & -19 & $-18(5)$ & $-19(7)$ & 1.03 & $(0.94-1.13)$ & 0.558 \\
\hline Basal & -18 & $-20(6)$ & $-24(9)$ & 1.09 & $(1.00-1.19)$ & 0.039 \\
\hline Medium & -20 & $-19(6)$ & $-19(9)$ & 1.01 & $(0.94-1.09)$ & 0.807 \\
\hline Apical & -19 & $-14(6)$ & $-12(8)$ & 0.96 & $(0.89-1.05)$ & 0.368 \\
\hline \multicolumn{7}{|l|}{$\operatorname{Sr}\left(s^{-1}\right)$} \\
\hline Basal & 0.70 & $1.93(1.00)$ & $2.34(0.86)$ & 0.61 & $(0.32-1.20)$ & 0.145 \\
\hline Medium & 0.85 & $1.44(0.51)$ & $1.63(0.69)$ & 0.67 & $(0.28-1.61)$ & 0.368 \\
\hline Apical & 0.86 & $1.27(0.10)$ & $1.30(0.56)$ & 0.87 & $(0.30-2.52)$ & 0.802 \\
\hline
\end{tabular}

FAC: Fractional Area Change, TAPSE: Tricuspid Annular Plane Systolic Excursion, S': Doppler-derived tricuspid lateral annular systolic velocity, LS: Longitudinal Strain, Sr: Strain Rate. 
Table 4. Multivariate analysis for PGD3 development.

$\begin{array}{lccccc} & \boldsymbol{\beta} & \text { S.E } & \text { OR } & \text { 95\% Cl } & \text {-value } \\ \text { Age (years) } & -0.003 & 0.028 & 0.10 & (0.94-1.95) & 0.906 \\ \text { Procedure type } & -0.321 & 0.599 & 0.73 & (0.22-2.35) & 0.592 \\ \text { Ischemia time (min) } & 0.005 & 0.005 & 1.01 & (0.10-1.02) & 0.310 \\ \text { sPAP (mmHg) } & 0.010 & 0.014 & 1.01 & (0.98-1.04) & 0.508 \\ \text { Basal LS } \geq \mathbf{- 2 1 . 5}(\%) & -1.517 & 0.683 & 4.56 & (1.20-17.38) & 0.026\end{array}$

$\beta$ : Regression Coefficient, SE: Standard Error, sPAP: Systolic Pulmonary Arterial Pressure, Basal LS: Basal Free Wall Longitudinal Strain. 
Table 5. Bivariate analysis of association of basal LS $\geq 21.5 \%$ with PGD3.

\begin{tabular}{|c|c|c|c|}
\hline & OR & $95 \% \mathrm{Cl}$ & p-value \\
\hline Unadjusted base model & 3.72 & $(1.11-12.45)$ & 0.033 \\
\hline \multicolumn{4}{|l|}{ Base model adjusted for: } \\
\hline Age (years) & 3.71 & $(1.09-12.55)$ & 0.035 \\
\hline Procedure type & 3.73 & $(1.11-12.54)$ & 0.034 \\
\hline Ischemia time (min) & 4.14 & $(1.18-14.48)$ & 0.026 \\
\hline sPAP $(\mathrm{mmHg})$ & 4.14 & $(1.17-14.65)$ & 0.027 \\
\hline
\end{tabular}

